\title{
ESPLENECTOMIA SUBTOTAL POR VIA LAPAROSCÓPICA EM CÃES
}

\author{
LAPAROSCOPIC SUBTOTAL SPLENECTOMY IN DOGS
}

\author{
Andy Petroianu, TCBC-MG; ; René Berindoague Neto²
}

\begin{abstract}
RESUMO: Objetivo: O objetivo deste trabalho foi determinar a exeqüibilidade, reprodutibilidade e segurança da esplenectomia subtotal por via laparoscópica e suas repercussões morfológicas, hematológicas, imunitárias e de depuração sangüínea, comparando esses dados com os obtidos na esplenectomia laparotómica. Método: Este estudo experimental foi conduzido de acordo com os princípios éticos de pesquisa experimental, propostos pelo Comitê de Ética em Experimentação Animal da UFMG. Quatorze cães adultos mestiços de ambos os sexos foram distribuídos em dois grupos $(\mathrm{n}=7)$ : Grupo 1 - Esplenectomia subtotal laparoscópica, preservando o polo superior suprido exclusivamente pelos vasos esplenogástricos; a secção esplênica e hemostasia foram realizadas utilizando um grampeador linear cortante laparoscópico com carga de $45 \mathrm{~mm}$ de extensão. Grupo 2 - Esplenectomia total, realizada por meio de laparotomia mediana. Depois de três meses, foram analisadas as alterações nos exames hematológico, imunitário e cintilográfico em todos os animais. Secções dos remanescentes esplênicos, fígado e linfonodos foram avaliadas histologicamente. Resultados: Todos os procedimentos transcorreram sem complicações, não havendo morte operatória e com perda sangüínea mínima. Todos os animais sobreviveram à operação durante os três meses de acompanhamento. Após esse período, houve redução da contagem de plaquetas $(\mathrm{P}<0,05)$ no Grupo 1 . O restante dos exames hematológicos permaneceu normal. Os remanescentes esplênicos apresentaram arquitetura semelhante ao baço normal. Não houve alterações histológicas nos linfonodos e fragmentos hepáticos. Conclusão: É possível e segura a realização da esplenectomia subtotal por via laparoscópica no cão (Rev. Col. Bras. Cir. 2006; 33(5): 305-310).
\end{abstract}

Descritores: Baço; Esplenectomia; Laparoscopia; Preservação de órgãos; Modelos animais.

\section{INTRODUÇÃO}

A busca de conhecimento sobre o baço tem sido um desafio há mais de três milênios. O conceito aristotélico de que o baço não é essencial à vida e de que sua remoção pode até melhorar o quadro clínico de diversas doenças alcançou a metade do século XX. Entretanto, ao longo dos últimos anos, a esplenectomia total vem sendo substituída por procedimentos conservadores. Essa mudança ocorreu devido à conscientização de que o baço é um órgão importante do sistema mononuclear fagocitário. ${ }^{1,2}$

Diversos estudos vêm mostrando a importância da função imunológica exercida pelo baço e as implicações negativas da esplenectomia no sistema de defesa orgânica. Os resultados evidentes de correlação da esplenectomia total com a maior susceptibilidade a infecções despertaram interesse científico para o baço como órgão de defesa, principalmente contra a sepse aguda ${ }^{3,4}$.

O baço também representa um grande reservatório de linfócitos e exerce importante papel na formação de anticorpos, em especial a IgM, e pela síntese de fatores de complemento, sendo responsável por $25 \%$ a $30 \%$ da função fagocitária do sistema mononuclear fagocitário ${ }^{5-10}$. Diante desses conhecimentos, a preservação esplênica passou a ser meta prioritária nas operações realizadas sobre o baço em que se possa manter algum tecido esplênico. Por essa razão, a esplenectomia parcial vem sendo realizada para um número de indicações cada vez maior ${ }^{11,12}$.

Nossas observações, a partir de 1979, de que o pólo superior do baço apresenta vitalidade após a ligadura do pedículo esplênico principal, contribuíram para novas abordagens cirúrgicas sobre o baço. Essa particularidade deve-se ao fato de o pólo superior do baço receber um segundo tipo de vascularização, independentemente do pedículo, proveniente dos vasos contidos no ligamento esplenogástrico. Esse tipo de ressecção foi denominado esplenectomia subtotal, em contraposição à esplenectomia parcial, que é descrita na literatura e na qual são preservadas as artérias segmentares do pedículo ${ }^{13,14}$. A esplenectomia subtotal já foi estudada em várias doenças ${ }^{15-33}$.

Desde 1991, várias afecções esplênicas vêm sendo tratadas pela esplenectomia laparoscópica, considerada um procedimento cirúrgico avançado devido ao complexo suprimento sangüíneo esplênico e sua estreita correlação anatômica com o estômago, cólon e pâncreas. Em virtude da eficácia e de seus resultados, a esplenectomia laparoscópica tornou-se uma modalidade segura no tratamento de várias desordens hematológicas, na ressecção de cistos e tumores benignos, bem como em traumas esplênicos menores ${ }^{34-37}$. Entretanto, ressecções esplênicas parciais por via laparoscópica ainda são incomuns ${ }^{37-45}$.

1. Professor Titular do Departamento de Cirurgia da Faculdade de Medicina - UFMG; Docente Livre da Faculdade de Medicina de Ribeirão Preto USP; Docente Livre da Escola Paulista de Medicina - UNIFESP; Doutor em Fisiologia e Farmacologia; Pesquisador IA do CNPq.

2. Professor Adjunto do Departamento de Cirurgia da Faculdade de Medicina das Ciências Médicas de Minas Gerais; Mestre e Doutor em Cirurgia pela UFMG.

Recebido em 25-02-2006

Aceito para publicação em 30-03-2006

Conflito de interesses: nenhum

Fonte de financiamento: CNPq/FAPEMIG

Trabalho realizado no Departamento de Cirurgia da Faculdade de Medicina da Universidade Federal de Minas Gerais. 
Seguindo uma linha de pesquisa sobre a cirurgia conservadora do baço do Departamento de Cirurgia da Faculdade de Medicina da UFMG, observou-se que a esplenectomia subtotal é factível não apenas em estudos com camundongos, ratos, cobaias, coelhos, gatos, cães e porcos, mas também em seres humanos ${ }^{15,16,18-33}$. O objetivo deste trabalho foi avaliar experimentalmente a exeqüibilidade da esplenectomia subtotal por via laparoscópica, preservando apenas o pólo superior irrigado exclusivamente pelos vasos esplenogástricos, e suas repercussões morfológicas, hematológicas, imunitárias e de depuração sangüínea comparando esses dados com os obtidos na esplenectomia laparotómica.

\section{MÉTODO}

Este estudo teve como modelo experimental quatorze cães mestiços adultos, de ambos os sexos, pesando entre 9,8 e $15,5 \mathrm{~kg}$. Todos os cães foram vacinados contra raiva e submetidos a exame sorológico para leishmaniose brasileira. $\mathrm{O}$ estudo foi aprovado pela Comissão de Ética do Departamento de Cirurgia da Faculdade de Medicina da UFMG e seguiu os princípios éticos de pesquisa experimental propostos pelo Comitê de Ética em Experimentação Animal da UFMG.

Antes de iniciar o procedimento cirúrgico, amostras de sangue foram coletadas para realização de hemograma, dosagem de plaquetas e imunoglobulinas ( $\operatorname{IgA}$ A, $\operatorname{IgM}$ e $\operatorname{IgG})$. A anestesia geral foi induzida com pentabarbital sódico a $3 \%$ (Hypnol ${ }^{\circledR}$ - Labotatório Cristália São Paulo, Brasil - 30mg/kg intravenoso) e os animais foram mantidos com oxigênio administrado através de tubo endotraqueal. Não foi administrado antibiótico ou outro medicamento. As operações foram realizadas em condições assépticas após tricotomia e anti-sepsia da parede abdominal.

Os cães foram distribuídos aleatoriamente em dois grupos $(\mathrm{n}=7)$ :

- Grupo 1 - Esplenectomia subtotal por laparoscopia - Os cães foram colocados em posição supina, com leve proclive e rotação direita. A punção da cavidade foi realizada com agulha de Veress através de uma incisão mediana $4 \mathrm{~cm}$ acima do umbigo. Um pneumoperitônio com $\mathrm{CO}_{2}$ a uma pressão máxima de $12 \mathrm{mmHg}$ foi criado e um trocarte de 10/11 mm introduzido para realização da laparoscopia. Sob visão direta, um segundo trocarte de $10 / 11 \mathrm{~mm}$ foi posicionado na mesma linha, $3 \mathrm{~cm}$ inferiormente o umbigo, sendo posteriormente substituído por outro de $18 \mathrm{~mm}$ para dar entrada ao grampeador. Outros dois trocartes de $5 \mathrm{~mm}$ foram inseridos, um $1 \mathrm{~cm}$ abaixo do apêndice xifóide e outro no flanco esquerdo, $2 \mathrm{~cm}$ medialmente à crista ilíaca ântero-superior .

Todos os vasos esplênicos distais incluindo o pedículo principal foram ocluídos com clipes e seccionados, até a identificação dos vasos esplenogástricos, que foram preservados.

Em seguida, trocou-se o trocarte de $10 / 11 \mathrm{~mm}$ por outro de $18 \mathrm{~mm}$ para dar entrada ao grampeador. Na linha de demarcação entre a superfície esplênica isquêmica desvascularizada e aquela que permanecia recebendo suprimento sangüíneo, posicionou-se o grampeador linear cortante EndoGIA 45mm. (Figura 1a) Dessa forma, realizou-se o disparo dos grampos, em duas fileiras, com dupla linha de grampeamento cada, transversais ao baço. Uma fileira grampeou o remanescente esplênico e a outra a extremidade da parte esplênica a ser retirada. Com o próprio grampeador, seccionou-se transversalmente o baço entre as duas fileiras de grampos. (Figura 1b)

O segmento distal do baço foi removido dentro um saco plástico através do trocarte de $18 \mathrm{~mm}$, cujo orifício da parede abdominal foi ampliado para $30 \mathrm{~mm}$, facilitando sua retirada.

- Grupo 2 - Esplenectomia total por laparotomia - Trinta minutos antes das operações, 1,5 $\mathrm{ml}$ de solução de enxofre coloidal marcada com $99 \mathrm{~m}$ tecnécio foi injetada por via endovenosa em uma das patas do animal, com dose correspondente a 110 megabecquerel $(\mathrm{MBq})$. O animal era anestesiado e, em seguida, realizou-se uma laparotomia mediana supra-umbilical. O baço foi exteriorizado e todos os seus vasos ligados com fio de seda 3-0. Além do baço, retirou-se
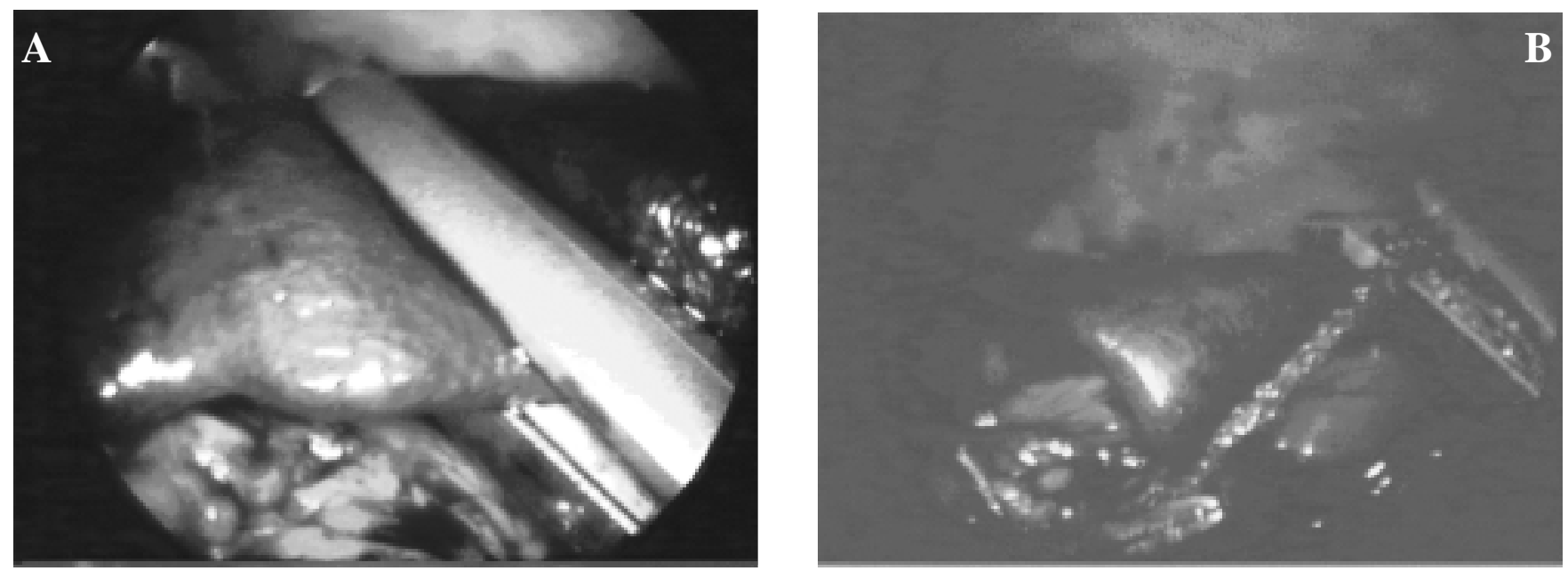

Figura 1 - Esplenectomia subtotal laparoscópica no cão. A - Grampeador posicionado para secção do baço. $\boldsymbol{B}$ - Baço seccionado pelo grampeador. Observe a borda do pólo superior esplênico com a linha de grampos, sem sinais de sangramento. 
um segmento em cunha da borda do lobo esquerdo hepático e um linfonodo do mesentério. Essas amostras (baço, fígado, linfonodo), juntamente com um coágulo sangüíneo, foram enviadas imediatamente para cintilografia. Após revisão da cavidade, a camada aponeurótica abdominal foi fechada com Vicryl ${ }^{\circledR} 2-0$, sutura contínua, e a pele com fio de náilon 4-0 em pontos separados. Em seguida, os cães foram acomodados em suas gaiolas.

Todos os animais foram acomodados em gaiolas individuais por um período de três meses, recebendo ração apropriada (Purina ${ }^{\circledR}$ - São Paulo - Brasil) e água à vontade. Decorrido esse período, uma solução de enxofre coloidal marcada com 99m tecnécio foi injetada via intravenosa, em todos os animais. Após trinta minutos, a morte dos cães foi induzida com tiopental sódico a 2,5\% (Thionembutal ${ }^{\circledR}-$ Lab. Cristália São Paulo - Brasil) , na dosagem de $50 \mathrm{mg} / \mathrm{kg}$, via endovenosa. Depois de mortos, retirou-se um fragmento de $2 \mathrm{~cm}$ da borda do lobo hepático esquerdo, um linfonodo mesentérico e coletados $10 \mathrm{ml}$ de sangue da veia cava inferior para formação de um coágulo sangüíneo. Nos animais do Grupo 1, além dessas amostras, o remanescente esplênico foi retirado para estudo.

Essas amostras foram enviadas para contagem cintilográfica. A análise da função fagocitária, por meio da captação do enxofre coloidal pelo método cintilográfico, foi estimada pelas contagens radioativas por grama de tecido. Cada amostra foi pesada imediatamente após a sua retirada. Levando-se em consideração o peso das amostras de fígado, baço, linfonodo e coágulo sangüíneo, a radioatividade da amostra foi calculada pela contagem de captação por grama de tecido. A quantidade total de radioatividade de todas as amostra recebeu um valor de "100". Com base nesse valor, as radioatividades dos órgãos foram proporcionais a 100.

Secções histológicas dos remanescentes esplênicos, fígado e linfonodo foram processados e corados com hematoxilina e eosina.
Os valores médios dos resultados foram estatisticamente comparados usando o teste $t$ Student. Valor de $P$ menor que 0,05 foi considerado nível de significância.

\section{RESULTADOS}

Todos os procedimentos transcorreram sem complicações, não havendo morte operatória e com boa recuperação pós-anestésica. Nenhum sangramento foi verificado, não sendo necessárias manobras adicionais ou outras medidas hemostasiantes. Em todos esses animais, o remanescente esplênico não apresentou sinais de isquemia aparente após o grampeamento. Na laparotomia realizada após três meses, todos esses remanescentes encontravam-se bem vascularizados e com aderências frouxas, principalmente ao omento, sem evidências de hemorragia subcapsular ou formação de abscesso. A linha de grampeamento do baço foi facilmente identificada. Nenhuma outra anormalidade abdominal foi encontrada.

A Tabela 1 mostra os resultados hematológicos, imunitários e cintilográficos em ambos os grupos. Anemia ocorreu em ambos os grupos no período pós-operatório. Redução da contagem de plaquetas $(\mathrm{P}<0,05)$ foi verificada no Grupo 1 . $\mathrm{O}$ restante dos valores médios permaneceram normais.

Depois de três meses, os remanescentes esplênicos apresentaram arquitetura semelhante ao baço. Em dois animais a secção histológica do remanescente mostrou uma pequena reação inflamatória inespecífica, com aumento de células polimorfonucleadas. Trabéculas foram verificadas dentro da polpa vermelha. A polpa branca mostrou aspecto normal. Não houve alterações histológicas nos linfonodos e fragmentos hepáticos.

\section{DISCUSSÃO}

O risco de sepse pós-esplenectomia tem estimulado a preservação de tecido esplênico e sua função. Os resulta-

Tabela 1 - Valores hematológicos, imunitários e cintilográficos no período pré-operatório e três meses após a esplenectomia subtotal laparoscópica (Grupo 1) e esplenectomia total (Grupo 2) nos cães.

\begin{tabular}{lccr}
\hline Parâmetros & Pré-operatório & Grupo 1 & Pós-operatório \\
& & 9.4 & Grupo 2 \\
\hline Hemoglobina (mg\%) & $13.6^{+}$ & 8180 & 9.0 \\
Leucócitos globais ( /il $)$ & 11300 & $80200^{+}$ & 12820 \\
Plaquetas (/ill) & 264000 & 122 & 202200 \\
IgM (mg/ 100ml) & 139 & 19 & 104 \\
IgA (mg/100ml) & 20 & 1321 & 23 \\
IgG $(\mathrm{mg} /$ / 100ml) & 1183 & 46.59 & 1115 \\
Fígado $(*)$ & 56.78 & 51.78 & \\
Baço $(*)$ & 42.67 & 0.81 & \\
Linfonodo $(*)$ & 0.35 & 0.83 & \\
Coágulo sangüíneo $(*)$ & 0.44 & & \\
\hline
\end{tabular}

Os valores hematológicos e imunitários da coluna pré-operatório foram a média dos valores de todos os cães dos grupos 1 e 2 juntos.

(*) Os valores médios dos exames cintilográficos foram considerados proporcionais à quantidade total injetada $=100$. Os valores presentes na coluna pré-operatório são relativos ao Grupo 2 (esplenectomia total)

$+=$ Diferença dos valores das outra duas colunas $(P<0.05)$ 
dos do presente trabalho confirmam nossos resultados prévios e mostram a possibilidade de preservar parte do baço quando os vasos esplênicos principais precisam ser ligados ${ }^{15-33}$. O uso de grampeadores mecânicos para realizar esplenectomia subtotal tem sido descrito recentemente ${ }^{34,35,42,45}$. Enquanto essa técnica é descrita em esplenectomia por laparotomia, ela ainda é pouco utilizada nas operações laparoscópicas.

Nossa experiência com a esplenectomia subtotal em hipertensão porta, trauma, doença de Gaucher, hepatoesplenomegalia mielóide, doenças na cauda pancreática, hipodesenvolvimento somático de origem esplenomegálica e na presença de leucemia linfocítica crônica mostraram ausência de partículas anômalas na amostras sangüíneas, sugerindo função dos macrófagos esplênicos. Essa possibilidade é confirmada por meio de imagens cintilográficas.

Sendo o baço o principal produtor de IgM do organismo, a medida de sua concentração sérica é uma das formas de acessar a função imunitária do tecido esplênico ${ }^{43,44}$ É pertinente supor que o remanescente esplênico contribui para a preservação das imunoglobulinas depois da esplenectomia subtotal.

A função esplênica pode ser rotineiramente estudada por métodos hematológicos, imunitários e cintilográficos. Investigação direta pode ser realizada por meio de imagens cintilográficas. O enxofre coloidal marcado com 99m tecnécio é um material usado para estudar o sistema mononuclear fagocitário, por ser fagocitado pelos macrófagos presentes no baço, permitindo um estudo quantitativo indireto ${ }^{6,7,22}$. Variações na radioatividade devido falhas metodológicas inerentes foram compensadas pela média proporcional dos resultados encontrados no presente estudo.

A captação do enxofre coloidal marcado com 99mtecnécio pelo remanescente esplênico, mostrou que a função fagocitária foi preservada, mesmo sem o pedículo vascular principal. Os resultados obtidos mostraram que o baço remanescente é capaz de fagocitar o enxofre coloidal marcado com o isótopo radioativo.

Para assegurar que os resultados do trabalho não sofreriam influência de afecção hepática, esplênica ou de outra parte do sistema mononuclear fagocitário, foram realizadas biopsias. De fato em animal algum foram verificadas alterações histopatológicas que interferissem neste trabalho.

Entretanto, deve-se considerar que o principal objetivo do presente trabalho foi mostrar que a esplenectomia subtotal é factível e segura pela via laparoscópica. A simplicidade da técnica cirúrgica com utilização de equipamento laparoscópico habitual e a ausência de complicações, confirmam a possibilidade de aplicação desse método em todas as esplenectomias enquanto não for possível manter o hilo vascular esplênico, e que seja permitido conservar tecido esplênico.

Por outro lado, é fundamental enfatizar que há diferenças anatômicas marcantes entre o abdome e o baço do homem e do cão. Enquanto o baço do homem é ovalado e restrito a uma pequena região próxima ao fundo gástrico, no cão ele é alongado e preenche toda a parte esquerda do abdome. A consistência esplênica do cão é maior e mais resistente do que a humana, além disso os vasos esplênicos caninos são nitidamente separados, enquanto os do baço humano estão localizados dentro dos ligamentos gastroesplênico e esplenorrenal. Portanto é muito mais fácil ligar ou clipar e seccionar os vasos do baço canino.

É possível e segura a realização da esplenectomia subtotal por via laparoscópica no cão. Os achados do presente trabalho não podem ser estendidos para a cirurgia em humanos sem reserva. Futuros estudos devem ser desenvolvidos a fim de verificar a importância de preservar o pólo superior esplênico. A esplenectomia subtotal laparoscópica deve ser considerada principalmente em pacientes imunossuprimidos, no trauma esplênico e em todos os casos em que o tecido esplênico puder ser conservado.

\section{AGRADECIMENTOS}

Os autores agradecem os auxílios do CNPq e da FAPEMIG para a compra do equipamento e de todo material utilizado na realização deste trabalho.

\footnotetext{
ABSTRACT

Background: The aim of this study is to determine the feasibility, reability and safety of the subtotal splenectomy performed by laparoscopic means, comparing these data with those obtained by mean of laparotomic splenectomy. Methods: An experimental study was conduced in accordance with the principles of the International Guiding for Biomedical Research Involving Animals. Fourteen adult mongrel dogs of both sexes were divided into two groups $(n=7)$ : Group 1 Laparoscopic subtotal splenectomy, preserving the upper pole supplied by splenogastric vessels; the splenic section, haemostasis and closure was performed with a 45 mm linear laparoscopic surgical stapler. Group 2 - Total splenectomy was performed through a median laparotomy. After three months, we accomplished haematological, immunological and scintigraphic exams in all animals. Sections of the remnant spleens, livers and lynph nodes were histologically processed. Results: Blood loss was minimal and all animals survived to operation and during the three months follow-up. Reduction $(P<0.005)$ of the platelets was verified in the Group 1. The rest of the haematological mean values remained normal. The remnant spleen, liver and lymph nodes showed normal histological aspects, with increasing amounts of polymorphonuclear cells observed in both groups. Conclusion: It is feasible and safe to perform subtotal splenectomy by laparoscopic means in dog.
}

Key words: Spleen; Splenectomy; Laparoscopy; Organ preservation; Models, animals. 


\section{REFERÊNCIAS}

1. Doan CA. The spleen: its structure and functions. Postgrad Med. 1968;43(5):126-31.

2. Saba TM. Physiology and physiopathology of the reticuloendothelial system. Arch Int Med. 1970;126(6):103152 .

3. King H, Shumacker HB Jr. Susceptibility to infection after splenectomy performed in infancy. Ann Surg. 1952;136(2):23942.

4. Singer DB. Postsplenectomy sepsis. Perspect Pediatr Pathol. 1973;1:285-311.

5. Llende M, Santiago-Delpín EA, Lavergne J. Immunobiological consequences of splenectomy: a review. J Surg Res. 1986;40(1):8594.

6. Petroianu A, Simal CJR, Barbosa AJA. Assessment of phagocytic function in remnants of subtotal splenectomy and in autologous spleen implantation. Med Sci Res. 1993;21:715-7.

7. Araújo ID, Petroianu A, Simal CJR, et al. $99 \mathrm{mTc}$ sulfur colloid uptake by rat liver, spleen and lungs in early biliary obstruction. Med Sci Res. 1996;24: 245-6.

8. Thalhamer J, Leitner W, Kurz ME, Liaunigg A, Seifriedsberger M, Bergmann ES, Kaindl H, Pimpl W. Immunoarchitecture and specific functions of splenic autotransplants at different implantation sites. Eur Surg Res. 1992;24(1):22-36.

9. Lüdtke FE, Mack SC, Schuff-Werner P, Voth E. Splenic function after splenectomy for trauma. Role of autotransplantation and splenosis. Acta Chir Scand. 1989;155(10):533-9.

10. Poulin EC, Thibault C, DesCöteaux JG, Cote G. Partial laparoscopic splenectomy for trauma: technique and case report. Surg Laparosc Endosc. 1995;5(4):306-10.

11. Keramidas D, Buyukunal C, Senyuz O, Dolatzas T. Splenic artery ligation: a ten-year experience in the treatment of selected cases of splenic injuries in children. Jpn J Surg. 1991;21(2):1727.

12. Allendorf JDF, Bessler M, Kayton ML, Oesterling SD, Treat MR, Nowygrod R, Whelan RL. Increased tumor establishment and growth after laparotomy vs laparoscopy in a murine model. Arch Surg. 1995;130(6):649-53.

13. Petroianu A, Petroianu S. Anatomy of splenogastric vessels in patients with schitosomal portal hypertension. Clin Anat. 1994;7(1):80-3.

14. Campos Christo MB, DiDio JL. Anatomical and surgical aspects of splenic segmentectomies. Ann Anat. 1997;179(5):461-74.

15. Petroianu A. Esplenectomia subtotal e anastomose esplenorenal proximal para o tratamento da hipertensão portal. Rev Bras Cir. 1983;73(2):101-4.

16. Petroianu A. Estudo quantitativo da função fagocitária de macrófagos em baços íntegros e em remanescentes de esplenectomia subtotal, em mamíferos [dissertação]. Ribeirão Preto (SP): Universidade de São Paulo; 1993.

17. Petroianu A. Subtotal splenectomy and portal variceal disconnection in the treatment of portal hypertension. Can J Surg. 1993;36(3):251-4.

18. Petroianu A. Treatment of cystadenoma of the pancreatic tail by distal pancreatectomy and subtotal splenectomy. Dig Surg. 1995;12:259-61.

19. Petroianu A. Subtotal splenectomy for treatment of patients with myelofibrosis and myeloid metaplasia. Int Surg. 1996;81(2):177-9.

20. Petroianu A. Subtotal splenectomy in Gaucher's disease. Eur J Surg. 1996; 162(6):511-3.

21. Petroianu A. Treatment of portal hypertension by subtotal splenectomy and central splenorenal shunt. Postgrad Med J. 1988;64(747):38-41.
22. Petroianu A, Barbosa AJA. Quantitative studies on macrophage phagocytosis in whole spleen and in the remnant of subtotal splenectomy. Med Sci Res. 1991;19:373-5.

23. Resende V, Petroianu A. Subtotal splenectomy for treatment of severe splenic injuries. J Trauma. 1998;44(5):933-5 .

24. Petroianu A, Silva KDA. Esplenectomia subtotal para tratamento do hipodesenvolvimento somático e sexual secundário a esplenomegalia. Rev Col Bras Cir. 2002;29(4):209-12.

25. Petroianu A. Subtotal splenectomy for treatment of retarded growth and sexual development associated with splenomegaly. Minerva Chir. 2003;57(3):413-4.

26. Petroianu A. Avaliação da função fagocitária em remanescentes de esplenectomia subtotal e auto-implante esplênico autógeno Rev Bras Hematol Hemoter. 2003;25(1):25-31.

27. Petroianu A. Tratamento cirúrgico da hipertensão porta na esquistossomose mansoni. Rev Soc Bras Med Trop. 2003;36(2):253-65.

28. Petroianu A. O Baço. São Paulo: CLR Balieiro; 2003.

29. Resende V, Petroianu A. Funções do remanescente esplênico após esplenectomia subtotal ou auto-implantes esplênicos para tratamento de lesões graves do baço humano. Rev Med Minas Gerais. 2001;11(1):59.

30. Resende V, Petroianu A. Funções do remanescente esplênico após esplenectomia subtotal para o tratamento de lesões complexas do baço humano. Rev Assoc Med Bras. 2002;48(1):26-31.

31. Resende V, Petroianu A. Functions of the splenic remnant after subtotal splenectomy for treatment of severe splenic injuries. Am J Surg. 2003;185(4):311-5.

32. Petroianu A. Subtotal splenectomy for the treatment of chronic lymphocytic leukemia. Ann Hematol. 2003;82(11):708-9. Epub 2003 Aug 2.

33. Petroianu A, De Oliveira AE, Alberti LR. Hypersplenism in schistosomatic portal hypertension. Arch Med Res. 2005;36(5):496-501.

34. Delaitre B, Maignien B. Splénectomie par voie coelioscopique. Presse Med. 1991;20(44):2263.

35. Carroll BJ, Phillips EH, Semel CJ, Fallas M, Morgenstern L. Laparoscopic splenectomy. Surg Endosc. 1992;6(4):183-5.

36. Targarona EM, Espert JJ, Balagué C, Piulahs J, Artigas V, Trias M. Splenomegaly should not be considered a contraindication for laparoscopic splenectomy. Ann Surg. 1998;228(1):35-9.

37. Katkhouda N, Mavor E. Laparoscopic splenectomy. Surg Clin North Am. 2000;80(4):1285-97.

38. Feliciotti F, Sottili M, Guerrieri M, Paganini AM, Lezoche E. Conservative ultrasound-guided laparoscopic treatment of posttraumatic splenic cysts: report of two cases. Surg Laparosc Endosc. 1996;6(4):322-5.

39. Mayberry JC, Sheppard BC, Mullins RJ. Laparoscopic management of an enlarging subcapsular splenic hematoma. J Trauma. 1998;44(3):565-7.

40. Linhares MM, Caetano EM, Louzano R, Sakamoto FI, Cruz OM, Lopes Filho GJ. Surgical resection of traumatic spleen cysts by laparoscopy. Int Surg. 1998;83(4):308-10.

41. Seshadri PA, Poulin EC, Mamazza J, Schlachta CM. Technique for laparoscopic partial splenectomy. Surg Laparosc Endosc Percutan Tech. 2000;10(2):106-9.

42. Corcione F, Cuccurullo D, Caiazzo P, Settembre A, Bruzzese G, Vittoria I, Cusano T. Laparoscopic partial splenectomy for a splenic pseudocyst. Surg Endosc. 2003;17(11):1850.

43. Sapucahy MV, Faintuch J, Bresciani CJC, Bertevello PL, HabrGama A, Gama-Rodrigues JJ. Laparoscopic versus opern splenectomy in the management of hematologic diseases. Rev Hosp Clin Fac Med S Paulo. 2003;58(5):243-9. 
44. Uranüs S, Pfeifer J, Schauer C, Kronberger L, Rabl H, Ranftl G, Hauser H, Bahadori K. Laparoscopic partial splenic resection. Surg Laparosc Endosc. 1995;5(2):133-6.

45. Uranüs S, Kronberger L, Kraft-Kine J. Partial splenic resection using the TA-stapler. Am J Surg. 1994;168(1):49-53.

Como citar este artigo:

Petroianu A, Berindoague Neto R. Esplenectomia subtotal por via laparoscópica em cães. Rev Col Bras Cir. [periódico na Internet] 2006 set-Out; 33(5). Disponível em URL: http://www.scielo.br/rcbc
Endereço para correspondência:

Prof. Andy Petroianu

Avenida Afonso Pena, 1626 apto. 1901

30130-005 - Belo Horizonte - MG

Fone / Fax: (31) 3274-7744

E-mail: petroian@medicina.ufmg.br 